\title{
Grounding and metaphysical explanation: it's complicated
}

\author{
Anna-Sofia Maurin ${ }^{1}$ (D)
}

Published online: 17 March 2018

(C) The Author(s) 2018

\begin{abstract}
Grounding theorists insist that grounding and explanation are intimately related. This claim could be understood as saying either that grounding 'inherits' its properties from (metaphysical) explanation (and that, therefore, contemplating the nature of explanation informs us about the nature of grounding) or it could be interpreted as saying that grounding plays an important - possibly an indispensablerole in metaphysical explanation (and that, therefore, that there are these explanations justifies positing grounding). Or both. I argue that saying that grounding 'inherits' its properties from explanation can only be justified if grounding is explanatory by nature (if so-called 'unionism' is true), but that this view is untenable. We ought therefore to be 'separatists' and view grounding and explanation as distinct. As it turns out, though, once grounding has been in this sense distinguished from the explanation it backs, the view that the role grounding plays in explanation justifies its introduction ends up in serious trouble. I conclude that the role grounding plays in explanation (if any) does not justify attributing to grounding whatever nature we think it has, and it most likely does not give us any special reason to think grounding exists.
\end{abstract}

Keywords Metaphysical explanation · Grounding · Justification · Understanding

\section{Introduction}

Assume, at least for the purposes of the argument set out here, that there is such a thing as grounding. Perhaps more controversially, assume that, when formulating a view of its nature, we are trying to uncover rather than construct it. Grounding, if it

Anna-Sofia Maurin

anna-sofia.maurin@gu.se

1 Department of Philosophy, Linguistics and Theory of Science, University of Gothenburg,

Gothenburg, Sweden 
exists, is out there to be discovered. ${ }^{1}$ Then according to the 'orthodoxy' grounding is a hierarchical dependence-relation ${ }^{2}$ that holds between worldly facts or states of affairs. ${ }^{3}$ More precisely, it is an objective and mind-independently obtaining hyperintensional and non-monotonic strict partial ordering relation-asymmetric, irreflexive, and transitive-always holding between what is less and what is more fundamental (such that the more fundamental grounds the less fundamental, but not vice versa). Or something to that effect. For, even if this way of characterizing grounding is the orthodoxy, it is still very much contested. None of the features just listed have gone unquestioned and hardly any grounding theorist thinks grounding has all of them. One feature-that grounding is explanatory-does not fit this mold. This is a feature that (probably) all grounding theorists think characterizes grounding and one that most believe plays an important role in distinguishing grounding from other types of metaphysical dependence. This is a paper about that feature. ${ }^{4}$

\subsection{Metaphysical explanation}

Common to most (perhaps all) expressions of the thought that grounding is explanatory is the idea that it is so in a 'special' way. Sometimes this thought is further spelled out by saying that grounding is or backs (I'll come back to this disjunction below) metaphysical explanation. Exactly what a metaphysical explanation is, is mostly left somewhat vague. Minimally, most seem to agree, it is an explanation which accounts for the nature and/or existence of something with reference to something else on which the first thing non-causally and synchronically depends. Something which somehow (again, non-causally and synchronically) determines or makes the second thing exist and be the way it is. ${ }^{5}$

\footnotetext{
1 This is hence a paper on what Dasgupta (2017) calls inflated ground. One reason I choose to investigate this, rather than its deflated cousins, is that some philosophers obviously operate with such a notion, and that if you do, grounding's relationship with explanation becomes an especially interesting yet thorny affair. Another reason is that I think inflated grounding, in being more substantial, is also more interesting. If we can make good sense of it, we can also make important use of it.

${ }^{2}$ Whether grounding is a relation has been debated. Arguments on either side have mostly been put in terms of what is the 'best' way to linguistically represent grounding. Proponents of the relational view have argued that grounding statements featuring the relational predicate 'grounds' most correctly depict the world they're about (Rodriguez-Pereyra 2005; Trogdon 2013). But grounding can also be picked out using the sentential connective 'because' or the sentence-forming operator 'in virtue of'. Whether those who argue that grounding is not best represented using a relational predicate are in fact opposed to the idea that grounding is a relation is unclear. For, mostly, those people tend to prefer alternative ways of representing grounding because of their supposed 'ontological neutrality' (Correia 2010; Fine 2012).

3 Not everyone thinks the relata of grounding must be states of affairs. According to e.g., Schaffer 2009, grounding can hold between all sorts of things. And if truthmaking is a species of grounding, grounding holds cross-categorically.

${ }^{4}$ Nothing I will say depends on my adopting the convention of calling the relation which holds between worldly states of affairs grounding, and the explanation it is or backs metaphysical explanation. Alternative labels for the former include construction-, production- or building-relation, and for the latter constitutive-, in-virtue-of- or compositional explanation.

5 As this is only a first sketch of an account of the nature of metaphysical explanation, it stands in need of both refinement and adjustment. If e.g., A. Wilson (forthcoming) is right, and the relevant dependence
} 
With this first stab account of metaphysical explanation in mind, the following explanations - all found in metaphysics ${ }^{6}$ - seem to fit the bill:

i. Singleton-Socrates exists because Socrates exists.

ii. Socrates exists because a certain bundling of tropes exists.

iii. Wisdom exists because the class of all wise things exists.

In fact, areas of philosophy mostly understood as outside metaphysics seem likewise ripe with metaphysical explanation:

iv. Sentences have the meanings they do because they serve certain social and/or biological functions.

v. Consciousness exists and has the nature it does because of the existence and nature of certain forms of brain activity.

vi. A thing's moral properties exist and are the way they are because that thing's physical properties exist and are the way they are.

vii. Gender, race and class exist because of the existence of the collective intentions and actions of certain groups.

Here, just as in (i)-(iii), something (the meaning of a sentence, consciousness, a thing's moral properties, or the existence of gender, race and class) is explained by something else (a sentence's social and/or biological function, brain activity, a thing's natural properties, or a group's collective intentions and actions) on which the first thing non-causally and synchronically depends. Indeed, metaphysical explanation seems a far from uncommon phenomenon also outside philosophy. Why is the water acidic? Interpreted as a question about the causal origins of the water's acidity, a suitable explanation refers to the splash of lemon juice that was just added to it. Interpreted non-causally, an equally suitable explanation refers to the number and configuration of hydrogen ions that contribute to the water's makeup. This arguably generalizes. Every time we ask for an account of the nature and/or existence of some macro-phenomenon, a possible explanation is one that refers to the micro-phenomena on which that macro-phenomenon non-causally and synchronically depends. Whether these are all explanations of the sort envisaged by the grounding theorist will depend on whether the non-causal making or determining of invoked in the explanation is grounding. For now, let's assume that it is.

\footnotetext{
Footnote 5 continued

relation could be diachronic, our account of the nature of metaphysical explanation would need to be reformulated in a way that reflects this.

${ }^{6}$ Metaphysical explanation is not the only kind of explanation you find in metaphysics. Metaphysical reasoning sometimes proceeds via causal explanation. It also involves explanation more in the sense of modeling. Explanations thus understood are weighed against each other and the 'best' explanation is chosen based on its superior virtue (Paul 2012). Metaphysical explanation of the kind that interests us here is more narrowly concerned with what grounds the existence and/or nature of some specific $x$ (although, certainly, explanation thus understood will feature prominently in explanation of the modeling-kind).
} 


\subsection{Inheritance, involvement, informativeness and justification}

Two things grounding theorist have said (or at least implied) about grounding and explanation seem especially well equipped to serve as explications of the thought that grounding and explanation are somehow intimately related:

Inheritance grounding 'inherits' (some of) its properties from explanation.

Involvement (metaphysical) explanation is such that grounding plays a-possibly indispensable-role in it.

Take Inheritance first. Many grounding theorists, when pressed on why they think grounding has the properties it does, point out that this is because those are properties had by explanation. It is because explanation is void if circular, that grounding is asymmetric, and it is because explanation in part consists in the supplying of 'new' information, that grounding is irreflexive (Trogdon 2013: 106). It is because in an explanation, only what is relevant to account for the explanandum features in the explanans, that grounding is non-monotonic (Rosen 2010: 116; Trogdon 2013: 109; Dasgupta 2014: 4). It is because substitution salva veritate destroys explanation, that grounding is hyperintensional (Kim 1993: 167; Schaffer 2009: 364). And it is because explanation must relate facts or states of affairs that grounding does too (Clark and Liggins 2012: 819; Trogdon 2013: 105; Raven 2013: 195). Even when there is disagreement over which properties characterize grounding, this is sometimes because there is disagreement over which properties characterize explanation. So, for instance, is irreflexivity under fire if selfexplanatory states of affairs are possible. Asymmetry is in trouble if one allows for circular explanation (Bliss 2014). And the ordinary epistemic virtues (like that of affecting — by increasing—our understanding) arguably clash with transitivity. ${ }^{7}$ For an unusually clear statement of this sort of attitude, here is Raven (2013: 193-194; $c f$. also his 2015):

[The] raison d'être for ground [i.e., to provide explanations of the less in terms of the more fundamental] imposes distinctive constraints on ground's logic, such as (i) irreflexivity: just as nothing explains itself, nothing grounds itself; (ii) transitivity: just as the explanation of an explanation also explains the explanandum, so too the grounds of the grounds of the grounded also ground the grounded; (iii) asymmetry: just as cyclical explanations are prohibited, so too are cycles of ground [...]; and (iv) non-monotonicity: just as explanation needn't survive arbitrary additional premises, so too ground needn't survive arbitrary additional grounds.

\footnotetext{
7 Other examples include: it being a well-founded relation (Cameron 2008 thinks it is, and Bliss 2013 thinks it isn't, in both cases because of how they conceive of the nature of explanation); it being transitive (Raven 2013 thinks it is because explanation is, but Daly 2005 argues that explanation is essentially intransitive, in which case grounding is too). Cf. also deRosset (2010) and Rodriguez Pereyra (2015).
} 
Involvement, next, is arguably universally accepted among grounding theorists. This is the idea that grounding is explanatory in the sense that it plays an importantperhaps an indispensable-role in explanation. It is often adopted alongside Inheritance but, as we shall see, it does not have to be. Here is Involvement as expressed by Audi (2012a: 708-709):

I posit grounding [...] as necessary to account for the correctness of certain explanations that cannot be understood as causal explanations. The function of grounding, then, is to make sense [...] of a certain kind of explanatory structure.

An interesting thing about the claim that grounding and explanation are intimately related is the use to which it has been put in arguing, both for the existence of grounding and for a specific way of getting to know its nature:

Informativeness that grounding resembles explanation informs us about the nature of grounding.

Justification that there is metaphysical explanation justifies positing grounding.

Informativeness is very naturally paired with Inheritance: if grounding is 'like' explanation, we learn things about its nature by contemplating the more familiar and easily accessible nature of explanation. And Justification is equally naturally paired with Involvement: if 'grounding-involving' explanation exists, then this seems like a very good reason to think grounding does too. Kovacs (2017) dubs what I call Justification 'the argument from explanatoriness' and considers it the master argument in favor of the existence of grounding.

This paper investigates the role explanation supposedly plays, both in characterizing and in justifying grounding. It does so via an investigation of the Inheritance/Informativeness claims (Sect. 2), followed by an investigation of the Involvement/Justification claims (Sect. 3). Its conclusions are mostly negative: grounding's relationship with explanation does not enlighten us about its nature, and it does not give us a reason-at least not a very strong one-to think grounding exists.

\section{Inheritance and informativeness}

To say that grounding and explanation are intimately related in the sense of Inheritance is to say two things. First, it is to say that grounding and explanation have (many of) the same properties. Second, it is to say that, that they do is no coincidence. Rather, that explanation has whatever properties it does gives us reason-it justifies-our thinking that grounding does too. In this, grounding is different from e.g., other strict partial ordering relations. Although they too share some of their properties with explanation-more precisely the formal properties of being irreflexive, asymmetric and transitive-the fact that explanation has those properties (assuming it does) does not give us any special reason to think that they do too. Most grounding theorists also assume that the nature of explanation is 
familiar and hence readily available to us. Thinking that it is, and that, therefore, so is the nature of grounding, is to adopt Informativeness.

\subsection{Unionism versus separatism}

Understanding the relationship between grounding and explanation in terms of Inheritance and Informativeness immediately gives rise to two questions:

1. What are the (relevant) properties of explanation?

2. Why think that those are properties explanation and grounding share?

As how we answer the second question arguably influences what we could or should say in response to the first, I will consider it first. In the literature, the relationship between grounding and explanation is-sometimes explicitly, but mostly implicitly-understood in either a 'unionist' or a 'separatist' sense (labels first introduced in Raven 2015). A 'separatist' is someone who thinks of grounding and (metaphysical) explanation as distinct. Grounding, on this view, backs explanation. Explanation tracks grounding. A 'unionist', on the other hand, has grounding and metaphysical explanation collapse. Grounding, on this view, is explanatory by nature. ${ }^{8,9}$

Inheritance is rarely on the agenda when the choice between separatism and unionism is debated, but I think it ought to be. For, if grounding and metaphysical explanation are distinct (i.e., if separatism is true) we have very little-arguably no-reason to think that contemplating the properties of explanation justifies attributing those same properties to grounding. The underlying reason for this is that saying of grounding that it is distinct from the explanation it backs, is most reasonably interpreted as saying something more than just that it is not identical to it. It is also to say that it and the explanation it backs belong to different kinds. More precisely, if grounding is a mind-independently obtaining worldly relation, adopting separatism amounts to saying of explanation that it is not a mind-independently obtaining and worldly relation. Rather, explanation is mind-involving, pragmatic, and/or 'epistemic' (whatever we take those locutions to mean more precisely). ${ }^{10}$ But then, as part of what it is to be an explanation is to be this mind-dependent and

\footnotetext{
${ }^{8}$ When I say that grounding-given separatism-is not explanatory 'by nature', I don't mean to imply that there is nothing in its nature that makes it apt to back (distinct) metaphysical explanation (cf. Sect. 3 below).

9 Pace Dasgupta, this is a substantive, not a merely verbal, distinction. That is, independently of what you choose to call what I call 'grounding' and 'metaphysical explanation', if your view is that there is a kind of explanation that tracks (distinct) worldly and non-causal dependence-relations, yours is a kind of separatism. According to Dasgupta, grounding (i.e., what we here call explanation) could, for all he knows, "be underwritten by production relations" (2017: 94, fn. 8). If it is, this would make him a separatist of sorts.

${ }^{10}$ It is of course perfectly possible to be a separatist yet view explanation as non-pragmatically constrained and objective (i.e., it is possible to hold that there are objective explanations which track mind-independent relations). It is however unclear why one would want to hold this view. For its proponent would arguably be left with all the disadvantages of being a unionist and none of the perks of being a ('normal') separatist. A raw deal indeed!
} 
epistemic thing, why think that explanation having the properties it does, justifies our thinking that those are properties had by worldly and mind-independent grounding? No good reason comes to mind. To be justified, the proponent of Inheritance ought hence to be a unionist.

Suppose unionism is true. Then grounding and metaphysical explanation are the same thing, and so it makes sense to say, both that they share properties, and that contemplating the properties of the one gives us reason to attribute them to the other. In fact, Leibniz' Law requires this! This means that we are now able to consider the first question: What characterizes (the relevant sort of) explanation? This is where our answer to the second question matters. For, if grounding is a worldly relation which obtaining or not is an entirely mind-independent affair, and if grounding is (metaphysical) explanation, metaphysical explanation is a worldly and mind-independently obtaining relation as well. ${ }^{11}$ But as we shall see, this is problematic. First, because if there is a kind of explanation that is in this sense entirely mind-independent, our general notion of explanation risks becoming so allencompassing as to become useless. And it is problematic, second, because even if there is a point to categorizing metaphysical explanation as 'an explanation', it is then an explanation so different from explanation in the ordinary sense, that what properties it, and hence grounding, has becomes somewhat of a mystery, thereby undermining Informativeness.

\subsection{What's in a name?}

That regarding grounding as explanatory by nature risks diluting our general notion of explanation, may at first glance seem unconvincing. This is because of the extremely variegated and heterogeneous uses we make of the notion of explanation anyway. 'Explanation' is an epithet given to such diverse things as 'mere' chunks of information, scientific theories, models, and speech-acts. Explanations, people say, are things we find, and things we give. They are context relative, and context independent. They are true or false, and they are more or less expedient. They are all-or-nothing, and they come in degrees. And so on. This is not necessarily because people hold contradictory beliefs about what an explanation is. Rather, 'explanation' is best understood as an umbrella term under which-sometimes radicallydifferent kinds of explanation fit. If grounding is explanatory by nature, then to the list of kinds of explanation belonging under the explanation-umbrella, we must add explanation in the sense of the holding of objective, mind-independent grounding relations. But then, if explanation - the umbrella term-is understood as something under which sometimes very different accounts of explanation fit, what harm is there in adding explanation in the unionist sense to the mix?

\footnotetext{
11 The unionist could of course opt instead for going in the other 'direction' and identify grounding with epistemic, mind-dependent explanation ( $c f$. e.g., Thompson 2016; Miller and Norton 2017; Dasgupta 2017). Although an interesting option, as this is a paper exploring the relationship between mindindependent_-inflated-grounding and explanation ( $c f$. fn. 1 for a very brief justification of this choice of focus), I will not consider it here.
} 
Here is one response that I find highly persuasive. Even though the explanationumbrella covers a wide range of different kinds of explanation, there seems to be one thing we take those explanations to share: an essential relation to understanding. ${ }^{12}$ Explanation is not just information. It is a function of the needs, knowledge, and expectations of those to whom the explanation is offered. It matters to (it ought to increase) the understanding of its (potential) receiver. Although it may or may not be understood as involving the obtaining of some worldly, mind-independent relation, therefore, explanation, most seem to agree, always involves something purely mind-dependent and epistemic. In Kim's words (1994: 54; $c f$. also e.g., Hempel 1965; Friedman 1974):

The idea of explaining something is inseparable from the idea of making it intelligible; to seek an explanation of something is to seek to understand it, to render it intelligible.

Of course, even if all explanations so-called up to this point have had a certain feature $F$, it does not follow that something could not be called 'an explanation' that doesn't have $F$. This is because of how our conceptualizations work. They evolve. They change. Everything is allowed. But then again, not everything is advisable. To illustrate, suppose you come up with a new dish, and you decide to categorize your dish as 'a dessert'. Then, either this is because your dish is like dishes most people would categorize as desserts or it is not. Suppose it is not. It seems excessive to demand that in cases like this, we must always introduce an entirely new category into which to stick our invention. In any case, this is not how we in fact categorize. Perhaps our general notion of a dessert at one point demanded of whatever fitted under it, that it did not contain meat. Yet now there are macarons flavored with foiegras and there is pig candy (true examples!). This is fine; we still know what a dessert is. But suppose my dish is inedible. Then categorizing it as 'a dessert' (or even as 'a dish') means changing our general notion of a dessert in a way that, although not impossible, is certainly not advisable. If 'being a dessert' is not supposed to signal things like 'is good to have after a meal' or 'is something you'll enjoy, although it might make you fat', it is not clear what use it is to us. Likewise, if grounding is explanatory by nature, metaphysical explanation is mind-independent. Therefore, if metaphysical explanation is 'an explanation', explanation is not essentially epistemic after all. But then it is not clear what saying of something that it is 'an explanation' is supposed to signal. Extending the domain of 'explanation' to include also explanation in the sense of the obtaining of worldly, mind-independent grounding relations, although possible, is therefore unadvisable.

\subsection{The threat to informativeness}

Arguing that unionism is untenable because it unduly dilutes our general notion of explanation is controversial. In part this is because, as we have seen, it is not clear

\footnotetext{
12 Which need not involve a sense of understanding but must involve a subject that thinks/represents or at least does things. Cf. e.g., Waskan (2006), Ylikoski (2009) and Khalifa (2013).
} 
what - even disregarding metaphysical explanation — should be allowed to fit under the 'explanation-umbrella' in the first place. In part, it is because the assumption that everything we might want to call 'an explanation' must share some essential feature(s) is controversial. Perhaps what makes something fit to be called 'an explanation' is more like what-according to Wittgenstein (1953)—makes something a game; in which case there need be no feature which all explanations share. It is controversial, finally, because even if there is some feature(s) which all explanations share, that this feature has to do with the role explanation plays in (increasing) understanding, or, more generally, that this feature is in any way 'epistemic', could be debated.

Suppose, therefore, that mind-independent grounding can be understood as a kind of explanation, without this threatening our substantive use of that categorization. Still there is trouble. According to Informativeness, because grounding 'inherits' its properties from explanation, studying the (readily available) properties of the latter, informs us about the nature of the former. If grounding is explanatory by nature-if unionism is true-it is however not clear what this means in practice. For in that case, explanation is something which obtains independently of whether there is someone there to grasp it and, crucially, independently of whether anyone's understanding is thereby at least potentially affected. This, we just saw, makes this a sort of explanation that is radically different from the kinds of explanation with which we are more familiar. Yet, when we let the properties of explanation guide us in our characterization of grounding, our ideas about what those properties are, arguably derive from intuitions formed based on our encounters with 'normal' explanation. Why do we think that explanation is non-monotonic? Because any part of an explanation should contribute to our understanding of that which it is an explanation of. Why do we think that explanation is irreflexive? Because in order to increase our understanding, an explanation must supply us with new information. And so on. We may disagree about individual examples, but the point remains. Ideas we have about the nature of explanation are based on experiences we have had of 'normal' explanation. Which means that, even if Inheritance is true (in the trivial sense that it would be true if grounding is explanation), Informativeness comes out as false. But then, as Thompson points out (2016: 397):

...we lose our grasp on what metaphysical explanation actually $i s$, and so it is very hard to see how the connection between ground and metaphysical explanation could shed light on grounding. The connection between grounding and explanation is only illuminating in so far as the relevant form of explanation is similar enough to our ordinary conception for us to understand it.

Inheritance, it seems, is in big trouble. It doesn't seem justifiable given separatism, and it seems to lead to all sorts of trouble given unionism. Is there a way out?

\subsection{A compromise view?}

One option is to deny what has so far been assumed: that if grounding is explanatory by nature, we lose every means to account for its relationship with understanding. 
Adopting the alternative - that explanation can be mind-independent yet somehow involve mind-dependent understanding - arguably amounts to adopting something along the lines of Michael Strevens' "simple view" (2008: 3, 2013: 510). ${ }^{13}$ According to the simple view:

An individual has scientific understanding of a phenomenon just in case they grasp a correct scientific explanation of that phenomenon.

On this view, understanding essentially involves explanation, yet explanation is separated from understanding, somewhat as, on a separatist view, explanation is separated from grounding.

The simple view can be 'translated' into our present context in at least two ways. On one interpretation, it is the view that explanation is worldly, mind-independent grounding - that unionism is true-and that understanding amounts to the minddependent and epistemic 'grasping' of mind-independent explanation. On another, it is the view that grounding, explanation, and understanding are all distinct-a version of separatism - and that understanding amounts to the mind-dependent 'grasping' of inherently epistemic explanation, which, in turn, tracks worldly and mind-independent grounding. I'm not going to debate the pros and cons of the simple view in either guise. I will only explain why I think that adopting the simple view does not help the proponent of Inheritance and Informativeness.

Note, first, that on the present view(s), although explanation is essential to understanding, understanding is not essential to explanation. This amounts to a separatism of sorts, even on the first-unionist-interpretation of the view. Which means that Informativeness becomes hard to justify. To see why that is, consider the first interpretation of the simple view first. On this view, explanation is mindindependent and worldly, which means that explanation is 'abnormal'. This is however not because explanation-given unionism-can have nothing to do with understanding. It can, precisely because understanding is understood as distinct from explanation. The proponent of this version of the simple view can even claim that on her view, intuitions about the nature of explanation (the intuitions that are supposed to inform us about the nature of grounding), rather than being based on our interactions with 'normal' explanation (which would be irrelevant), will be based on what we think is true of grasping. But this does not help, of course. For, just as 'normal' explanation and grounding are of different kinds, so are 'grasping' and grounding. Which means that we have no good reason to think that whatever characterizes the former also characterizes the latter. Consider the simple view in its second interpretation next. On this view, although explanation is separated from understanding, explanation remains epistemic (and hence at least somewhat 'normal'). But, again, this does not help. For explanation-as well as understanding-remain different enough from grounding to undermine our justification for Inheritance. The proponent of Inheritance - at least in its informative guise-has run out of options.

13 Thanks to Alexander Skiles for suggesting this option. 


\subsection{Moving on}

Nothing that has been argued in this section shows that grounding and explanation cannot be intimately related in the sense that they-because they are the same thing-have the same properties. Nor has it been shown that whatever properties explanation has cannot inform us about the properties of grounding. Rather, what has been argued is that, if grounding is explanatory by nature and if grounding is a worldly, mind-independent relation, then whatever properties it has, cannot be properties it inherits from 'normal' explanation. It can be properties it inherits from 'abnormal' explanation, but this doesn't really inform us about the nature of grounding. We know as little (or as much) about the nature of abnormal explanation, as we do about the nature of grounding. More importantly, saying that grounding inherits its properties from 'abnormal' explanation certainly doesn't justify attributing to grounding many of the properties it has according to the orthodoxy.

Suppose therefore that separatism is true. Then grounding and metaphysical explanation are distinct, which means that we can treat the latter as more or less 'normal' - essentially mind-involving —explanation. The price is Inheritance and Informativeness. If grounding and metaphysical explanation are distinct, and if explanation, but not grounding, is essentially mind-involving, we have no good reason to suppose that whatever properties grounding has, it has because those are properties had by the explanation it backs. We are still free to say that grounding and explanation are intimately related in the sense of Involvement, though. Whether this is enough for the grounding theorist who wishes to justify her posit with reference to its intimate relationship with explanation is the topic of the next section.

\section{Involvement and justification}

A good thing about being a separatist is that it allows you to treat explanation as a (mostly) epistemic phenomenon. This means that our understanding of specifically metaphysical explanation can now be modeled on an understanding of explanation with which we are (arguably) more familiar. Not any old understanding will do, of course. To adopt, say, a Deductive Nomological account ${ }^{14}$ ( $c f$. e.g., Hempel and Oppenheim 1948), means viewing explanation as a special kind of deductive argument. This view, along with e.g., pragmatist views of explanation, arguably fails to secure an important enough role for whatever worldly relation is being represented in the explanation (if any) for us to be able to make sense of Involvement and, consequently, of Justification.

\footnotetext{
14 The classic DN-model should not be confused with the account proposed by e.g., Wilsch (2015, 2016; cf. also Kment 2014). If, on the former view, mind-independently obtaining relations don't play an important enough role in explanation to support Involvement, the 'problem' with Wilsch's view is that, since it equates explanation with the obtaining of worldly mind-independent relations (plus the relevantworldly-metaphysical laws), it is a unionist rather than a separatist view, and hence open to the type of criticism set out in Sect. 2.
} 
If we want to be able to say that metaphysical explanation intimately involves mind-independently obtaining grounding relations, therefore, the account on which we model our understanding of metaphysical explanation must be realist. A good candidate is provided by $\operatorname{Kim}(1988,1994 ; c f$. also Salmon 1984). Kim distinguishes between explaining, having an explanation, and needing an explanation. The first, he tells us, is an epistemological activity, the second an epistemological achievement and the third is an epistemologically imperfect state one may be in. All three, in other words, lie on the side of knowledge; they are what Kim calls 'subjective'. Yet, since Kim is a realist about knowledge, worldly relations still play an important role in explanation. For, if knowledge involves truth, it also involves truth makers, which means that every bit of knowledge must have some objective counterpart that is not itself part of that piece of knowledge. According to Kim, more precisely, an explanation is a complex proposition-a purely representational item-divisible into explanans and explanandum $(C, E)$ such that, when we "have" an explanation, $E$ and $C$ stand in what Kim calls "the explanans relation" in our body of knowledge. Moreover, and crucially, $C$ is an explanans for $E$ (in part) in virtue of the fact that (non-representational) $c$ bears to $e$ what Kim calls "the explanatory relation": a determinate, objective and mind-independently obtaining relation $R$. That $c$ stands in $R$ to $e$ is then what the explanation "says". It is also what makes the explanation true (Kim 1988: 226).

The $R$ foremost in Kim's mind is of course causation, but it is clear (also to him) that other types of relations could play the $R$-role in (non-causal) types of explanation. Suppose, as seems likely, that grounding is one such relation. Does this mean that the role grounding would then play in specifically metaphysical explanation is a reason to think it exists? That depends. Either grounding is the only kind of relation apt to back metaphysical explanation, or it is not. If it is, then, clearly, the existence of metaphysical explanation strongly suggests that there is grounding. Is grounding the only relation apt to back metaphysical explanation? To be able to say-with justification-that it is, the proponent of grounding ought reasonably to be able to point to some feature(s) grounding has, and comparable non-grounding, non-causal relations lack, that makes it, but not them, uniquely explanation-apt. What if grounding is not the only relation apt to back metaphysical explanation? Then, to be able to support the existence of grounding with reference to the existence of metaphysical explanation, the proponent of grounding must come up with some reason for thinking that grounding is not always at least in principle substitutable by one of those other relations.

\subsection{Explanation-aptness}

That the existence of metaphysical explanation justifies positing grounding because grounding is uniquely apt to back such explanation is a common view among proponents of grounding ( $c f$. e.g., Fine 1995; Schaffer 2009; Clark and Liggins 2012; Raven 2013). Contrary to what one might perhaps think, to hold this view does not require that every (metaphysical) explanation we in fact give says that the relation it picks out is grounding. What the grounding theorist in favor of this view must say-what she wants to say-is rather that, even in cases where our 
explanation says it picks out some non-grounding, non-causal relation, the relation in fact picked out is grounding: for only grounding is apt to back metaphysical explanation.

To be justified, the proponent of this view must be able to point to some feature(s) that grounding has - and alternative relations lack - that makes it, but not them, uniquely explanation-apt. It is in other words not enough to simply stipulate that grounding is whatever is the worldly correlate of metaphysical explanation. Doing so would either beg the question against those who think that non-grounding, non-causal relations could do that job just as well, or it would at least fail to justify positing grounding understood as a relation that is distinct and different from alternative non-causal relations.

What, then, distinguishes grounding from comparable relations? The literature is replete with suggestions, most of which should by now be familiar. Grounding, but not these other relations, is explanation-apt because it, but not they, has 'drive', 'oomph', determination and direction. Only it, but not they, takes us from what is more to what is less fundamental. Only it is hyperintensional, and/or nonmonotonic, and/or asymmetric, and/or irreflexive, and/or transitive. And so on. Mostly, that these are features which matter to the role a relation can play in explanation is illustrated by contrasting grounding with supervenience. Supervenience, unlike grounding, lacks direction, primarily because it, again unlike grounding, is understood in purely modal terms. Thus understood, so goes the argument, it manages to do nothing more than to indicate certain patterns of (necessary) co-variation but fails to tell us anything about why these patterns hold, or about the nature of the dependence in question. Grounding, on the other hand, is directional, and so identifies the reason why e.g., Socrates and singleton-Socrates necessarily coexist: that singleton-Socrates exists in virtue of the existence of Socrates (Cameron 2016: 383). But even if all of these turn out to be good reasons to suppose that grounding, but not supervenience, is explanation-apt, pointing this out is not enough to justify the claim that grounding is uniquely apt to back metaphysical explanation. For, if only grounding backs metaphysical explanation, it must differ from every comparable metaphysical dependence relation, including (but not necessarily restricted to) what J. Wilson (2014) calls 'the small-g relations'. ${ }^{15}$ In fact, finding a difference is not enough. To be justified, the grounding theorist must also come up with some reason for thinking that this difference-whatever it is-matters to a relation's ability to back explanation. Living up to this brief is not an easy task.

According to most people engaged in this debate, all (or most) of the features mentioned in the previous paragraph, make grounding but not comparable relations explanation-apt precisely because those are features it shares with explanation. Yet

\footnotetext{
15 Wilson (2014) mentions type/token identity, functional realization, classical mereological parthood, the causal composition relation, the set membership relation, the proper subset relation, and the determinate/determinable relation. Trogdon (forthcoming) also includes set-formation and constitution on his list of (what he calls) determination-relations. Exactly what could or should count as a small-g relation is an interesting yet difficult question that, as we shall see in a moment, has some bearing on the issue at hand.
} 
the claim that this is what makes grounding uniquely explanation-apt cannot be justified. For, either this resemblance is a mere coincidence (somewhat as most assume that the proper parthood-relation - another strict partial ordering relationonly coincidentally resembles explanation) or it is not. Saying that it is not, is tantamount to (re-)invoking Inheritance. But as we have seen, if grounding is distinct from the explanation it backs, we have no good reason to think Inheritance is true, which means that we have no good reason to think that, because Inheritance is true, grounding is uniquely explanation-apt. And, obviously, if the similarity is merely coincidental, it cannot be invoked to help justify Justification.

To avoid these difficulties, the grounding-theorist might want to explore comparing grounding with another relation, often taken to stand in an analogously intimate relationship with explanation: causation. That grounding and causation have a lot in common is a view that has been proposed and explored by e.g., Schaffer (2016a) and A. Wilson (forthcoming). ${ }^{16}$ The similarities, they point out, are striking. Just as causation always takes place against a background of causal generalization, grounding always takes place against a background of metaphysical generalization. For both grounding and causation, moreover, these generalizations seem not merely accidental, but backed by some "formative principles"-whether laws of nature or laws of metaphysics-which serve to unify them. Grounding and causation, most believe, are both asymmetric, irreflexive and transitive relations. They both 'drive' (in a 'generative', 'productive', or 'determinative' manner), either across levels or across time. Therefore, if causation, in virtue of its nature, is uniquely apt to back a certain type of explanation, this gives the grounding theorist reason to think that grounding, in virtue of its (analogous) nature, is uniquely apt to feature in metaphysical explanation.

Three things can be said against reasoning in this way. First, that grounding and causation may not be all that similar after all. Second, that, even if they are similar, this does not rule out that other types of non-causal relations resemble causation to a similar degree. And, third, that even if grounding and causation resemble each other, and even if they resemble each other significantly more than either resembles some comparable non-causal relation, it is not clear why we should regard this as anything but coincidental. Take the first two points first. According to Bernstein ( $c f$. also Koslicki 2016), although grounding and causation seem similar, "there are more differences than commonalities to be found in the tendrils of both, and ignoring these differences while straining for the parallels is methodologically misguided" (2016: 23). One such difference is this: although causation is essentially diachronic, grounding is a synchronic relation. This difference, Bernstein thinks, has been underappreciated, but it ought not to be. For it is because of this difference that, although it is a substantive and largely open question whether an effect must always succeed its cause, the same is not true of the 'order' in which grounds and that which they ground present themselves: if $x$ grounds $y, x$ must 'precede' $y$ in the sense that $x$ must be more fundamental than $y$. Relatedly, although causation can be

\footnotetext{
${ }^{16} C f$. also Trogdon (forthcoming) for a slightly different take on how grounding (and metaphysical explanation) resembles causation (and causal-mechanistic explanation).
} 
either indeterministic or deterministic, grounding is always deterministic. In fact, it is so in an unusually strong-necessitating-sense that disallows e.g., (the analogue of) preemption. Causation and grounding behave differently, moreover, when it comes to the possibility of transworld causation/grounding (transworld causation but not transworld grounding is generally rejected). They differ in that causation, but not grounding, may admit of degrees, in that causation, but not grounding, may be dispositional (on a powers-ontology), in that grounding, but not causation, might be backtracking, and in that causation by double prevention does not have a clear grounding analogue. And so on.

We may of course disagree about each one of these examples (in fact, many have). What we should take away from Bernstein's critique is therefore not necessarily that grounding and causation aren't similar, or that, even if they are, that they are more dissimilar (dissimilar in more respects) than they are similar. The important lesson rather concerns the dangers involved in reasoning by analogy. My sister and I are very similar: genetically, experientially (up to a certain point in time); gender-wise; culturally, etc. Yet, we are also very different: in temperament; experientially (after a certain point in time); even genetically (full siblings on average, share $50 \%$ of their genes out of those that vary among humans). Suppose I am uniquely apt to do a certain job. Then even though my sister and I are more similar to each other than we are to, let's suppose, any other human being, this doesn't necessarily tell me very much about my sister's aptitude for an analogous job. What's important is if the respects in which I and my sister happen to resemble, rather than the respects in which we happen to differ, are what matters to our being able to do the job in question. And crucially, to find this out, we need to consider more than our resemblance per se. Likewise, for grounding and causation. Even if it turns out that they-and only they-share a significant number of properties, whether those are the properties that make them uniquely 'explanation-apt' will depend on other things than their resemblance per se. But then we are back with the old problem of saying what those 'other things' are, without re-invoking Inheritance.

These problems do not go away if we regard grounding, not as another relation on a par with alternative small-g relations, but as the kind to which those small-g relations apt to back metaphysical explanation belong. For, either the small-g relations belong to the grounding-kind because of some feature(s) they all share, or not. If not, then this is presumably because, 'being of the grounding-kind' is simply shorthand for 'being tracked by metaphysical explanation', in which case, the sense of grounding the existence of metaphysical explanation commits us to isn't grounding in the sense intended by the grounding-theorist. In order for the existence of metaphysical explanation to justify positing grounding-the substantive and 'natural' kind-therefore, there must be some feature(s) all relations belonging to the grounding-kind share. According to Koslicki (2015, 2016), however, we have reason to think that there is not. Looking at the sorts of relations proponents of grounding have tagged as being 'of the grounding kind', she argues, there is positive proof at most for their being 'objectively similar' in some respects. Evidence, she points out, that is compatible with their (also) being 'objectively dissimilar' (to an equal or higher degree) (2015: 340-341). There is hence reason to doubt that these 
relations are unified enough to belong to the same (substantive) kind. In any case, even if there turns out to be something all small-g relations share that makes it reasonable to say that 'they are of the same kind', the grounding-theorist must still, and as before, provide some reason(s) for thinking that what makes those relations belong to the same kind is also what makes them uniquely explanation-apt. And we have no reason to think that this is in any way easier if grounding is a kind of relation, rather than just one relation among the rest.

\subsection{Non-substitutability}

Let us therefore consider instead the view according to which, that there is metaphysical explanation justifies positing grounding because grounding is aptalthough not uniquely so-to be tracked by metaphysical explanation. This is arguably a much weaker sense of justification than what proponents of grounding normally intend when they say that the existence of metaphysical explanation justifies positing grounding. Yet in light of the difficulties we have found with the stronger view, it may be our safest bet.

To be justified, the proponent of this view must come up with some reason for thinking that grounding, although not the only relation apt to back metaphysical explanation, is essentially involved in at least some instances of such explanations. Against this, e.g., J. Wilson (2014: 553), has argued that, "[g]aining even basic explanatory illumination about metaphysical dependence requires appeal to the specific relations [the 'small-g relations'] ... that are the typical focus of investigations into such dependence". To be able to play at least some role in metaphysical explanation, therefore, grounding must always be accompanied by at least one of those other relations. But this, Wilson argues, makes grounding in general superfluous and hence dispensable.

Pace Wilson, e.g., Cameron (2016) and Schaffer (2016b) point out that, when Socrates asks Euthyphro, "Is the pious loved by the gods because it is pious, or is it pious because it is loved by the gods?" (Plato, Euthyphro 10a), one of the points he is making is that sometimes what we are after is an explanation in terms of what is (more) fundamental, such that the more fundamental explains the less fundamental. But this is a type of explanation which arguably tracks something more than the 'mere' small-g relations which always (let's suppose) hold between whatever relata are being picked out by the explanation. For, none of the small-g relations is inherently directional. Nor does the direction they occasionally exemplify (if any) necessarily match that of more to less fundamental (Cameron 2016: 4). Therefore, even if every time there is grounding there is also some small-g relation, this does not mean that there is no work for grounding to do. Quite the opposite. Its job is to ensure that there is direction enough to back explanations of the Euthyphro-kind.

Suppose Schaffer and Cameron are right: (at least a sub-group of the) metaphysical explanations are of the Euthyphro-kind. Suppose, moreover, that grounding is the only inherently directional relation on offer. Does this mean that, that there are explanations of this kind justifies positing grounding? According to Wilson, it does not. For, although small-g relations alone cannot furnish us with the right sort of directed dependence, this does not mean that we cannot make do 
without grounding. Rather, she argues, all the work grounding does in this respect can be done with recourse to primitive absolute fundamentality (Wilson 2014: 558f). On this view, which entities are in the fundamental base is primitive, yet manages to fix the direction of priority for the relevant small-g relation. Neither Cameron nor Schaffer think adding primitive fundamentality to the mix constitutes an improvement over simply accepting grounding. For, although absolute fundamentality can be defined in terms of grounding, the opposite is not true. Therefore, even if primitive fundamentality could furnish the small-g-theorist with the directedness she craves, the theory she ends up with is theoretically less attractive than the alternative. I think Cameron and Schaffer are right: grounding is preferable to primitive fundamentality for theoretical reasons. But this is not enough to justify Justification. For as long as a combination of small-g relations and primitive fundamentality can back explanations of the Euthyphro-kind, that there are such explanations does not in and of itself give us a reason to think grounding exists.

Suppose instead that Wilson is right, and that, in all cases where some small-g relation backs metaphysical explanation, grounding can be dispensed of. Does this mean that the grounding theorist has no way of justifying her posit with reference to the role it plays in explanation? Again, not necessarily. According to e.g., Trogdon (forthcoming), even if most cases of metaphysical explanation are such that what is being tracked is a small-g relation, cases of 'bare grounding' (i.e., grounding in the absence of concomitant small-g relations) are possible. Putative examples-which are all of the logical/conceptual type-include:

viii. $<p \vee q>$ because $<p>$.

ix. $\quad<p \& q>$ because $\langle p>$ and $<q>$.

$\mathrm{x}$. $\quad<$ Don is a bachelor $>$ because $<$ Don is male $>$ and $<$ Don is unmarried $>$.

In cases like these, Trogdon argues, the usual small-g relations do not play any role in explanation. Indeed, this is true even in cases where small-g relations are in fact present. To illustrate this, Trogdon asks us to consider the following explanation: Socrates exists or singleton-Socrates has $P$ because Socrates exists. In this case, Socrates, a constituent of the ground, stands in a candidate small-g relation (setformation) to singleton-Socrates, a constituent of the grounded. Yet this is irrelevant to the explanation at hand. That the fact that Socrates exists explains the fact that Socrates exists or singleton-Socrates has $\mathrm{P}$ rather depends on something like (or identical to) the inference rule disjunction introduction. And this, Trogdon claims, is not a small-g relation.

Suppose he is right about that. ${ }^{17}$ Does this mean that, that there are explanations like (viii)-(x), justifies our thinking that grounding exists? There are several reasons to be a bit skeptical of the claim that it does. For, (viii)-(x) can only help substantiate the claim that grounding is sometimes indispensable, if the relation tracked by those explanations (supposing they are explanations) is grounding, and if the explanations themselves are metaphysical explanations.

\footnotetext{
${ }^{17}$ He doesn't have to be-an issue I'll return to towards the end of this section.
} 
Now, as we have seen, if anything sets grounding apart from comparable small-g relations, it is its inherent directionality: if $a$ grounds $b, a$ is more fundamental than, and hence 'metaphysically prior' to, $b$. In the logical/conceptual case too, the explanans is arguably 'prior' to the explanandum in some sense of that word. The question is, if this is priority in a metaphysical sense. According to e.g., Hofweber (2009: 270; $c f$. also Audi 2012b) it is not. To him, the relationship between a true disjunction and its true disjunct ought at most to be understood as one of asymmetrical logical dependence, and, in cases like (x), the relationship is one of conceptual asymmetric dependence or priority. According to Wilsch (2016: 19f.), what this difference boils down to, is a difference in the subject-matter of our putative explanations. Logical and/or conceptual complexity is complexity which pertains to our representations only. Metaphysical complexity and priority has to do with the reality represented. This is why it seems wrong to say that whatever relation the logical/conceptual explanation tracks is grounding and the explanation itself is metaphysical.

Again, suppose this is right. Then what are we to say about the following cases of putative metaphysical explanation ${ }^{18}$

xi. The Magic Mountain exists because certain facts about Thomas Mann obtain. xii. I'm human as opposed to a swampman because of my past causal history.

Unlike (viii-x), these explanations arguably pertain directly to reality and not (just) to our representations. Yet it is unclear which small-g relation they track, if any. According to Wilsch, first, if some $a$ 's metaphysically explain $b$, this is because the $a$ 's 'construct' or 'make up' the $b$. Yet, "while the existence of The Magic Mountain is fully grounded in facts about Thomas Mann, the novel is not constructed from the man" (2015: 3297). And according to Trogdon (forthcoming), if whatever we want to classify as a small-g relation must be non-diachronic, there does not seem to be any such relation available for the swampman explanation to track.

Are these then pure ground metaphysical explanations? Not necessarily. For although they, because they pertain directly to reality, are most likely best categorized as cases of metaphysical explanation, it is not entirely clear why we could not think of them as metaphysical explanations of the small-g tracking kind. This is because, just as it is difficult to justifiably pin down the nature of grounding, it is difficult to pin down the nature of whatever small-g relations there may be. All we know for sure is that they are relations apt to back metaphysical explanation. And this is saying very little. Accepting that (xi) and (xii) are metaphysical explanations certainly teaches us something about whatever such explanations track, if anything. More precisely, it teaches us that what such explanations track is not necessarily material constitution or construction, and that it is not necessarily synchronic. You may of course have independent reasons for thinking that the small-g relations must have this or that nature, reasons which entail that the above cases are cases of metaphysical explanation in the absence of small-g relations.

\footnotetext{
18 (xi) was first suggested in Wilsch (2015: 3297) and (xii) by A. Wilson (forthcoming). Cf. also Trogdon (forthcoming).
} 
Until such reasons have been provided, however, putative cases of pure ground metaphysical explanation remain putative. As such, they at best lend a very weak conditional support to the claim that grounding is sometimes indispensable.

\section{Summary and conclusions}

In this paper, I have argued that, if we have reasons to believe that grounding exists, these have most likely nothing to do with explanation. And if we have reasons to believe that grounding has this or that nature, this is not because explanation does too. More precisely, I've argued that, if saying of grounding that it is explanatory is interpreted as saying that grounding 'inherits' its properties from explanation, then the kind of explanation grounding is turns out to be radically different from explanation in the 'normal' mind-involving sense. This does not mean that grounding could not be explanatory in an 'abnormal' sense. It only means that learning that it is does not really inform us about its nature. More precisely, it means that, that grounding inherits its properties from explanation does not in itself justify attributing to grounding the properties that have traditionally been thought to characterize it.

Better then to think of the way grounding and explanation are related in separatist terms. On this view, grounding and explanation are distinct, yet such that the latter involves the former. Does the role grounding plays in-separate-explanation give us a reason to think it exists? I have argued that it most likely does not. If grounding is uniquely apt to back metaphysical explanation, something must make it so. Saying that this special aptitude is due to grounding's inherent similarity to the explanation it backs is no option. For this would amount to re-invoking Inheritance, a thesis that, given that grounding and explanation are distinct, cannot be justified. Pointing out that grounding resembles causation-another relation commonly held to play an essential role in explanation-does not help. For, even if grounding and causation resemble each other more than they resemble any comparative small-g relation, that the respects in which they resemble are what matters to the role they play in explanation must be justified. Given that appealing to grounding's (and causation's) resemblance with explanation is now off the table, it is unclear what such a justification could amount to.

If grounding is not uniquely apt to back explanation, on the other hand, we need to come up with some reason for denying that, every time an instance of grounding backs an instance of explanation, that same instance of explanation could have been equally well supported by some alternative non-grounding relation (plus absolutely fundamentality). Our best bet in this respect proved to be so-called 'pure ground' explanations (i.e., cases of putative metaphysical explanation in the absence of small-g relations). Most of these cases turned out to be cases of logical/conceptual priority. As such, they could at most provide the grounding theorist with reason to think that a very specialized type of grounding exists. However, the more likely interpretation of cases of logical/conceptual priority is that they are not cases of metaphysical explanation, and that the relation they track is not grounding. In the absence of some independent reason for thinking that small-g relations must have 
this-or-that nature, finally, putative cases of pure ground metaphysical explanation that are not logical or conceptual, remain putative. As such they can at best only weakly and conditionally support the claim that grounding is sometimes indispensable. A disappointing result from the point of view of the grounding theorist.

Does this mean that there is no reason to think that grounding exists? No. There are probably plenty of reasons to think grounding exists, one being its ability to-in a theoretically parsimonious way-hierarchically structure reality. Does it mean that we have no reason to think that grounding has this or that nature? Again, no. Possible routes to insight into the nature of grounding include generalizing from paradigmatic cases of ground, and/or understanding grounding in analogy with some other-more familiar-phenomenon (like e.g., generation, production or building). It might even turn out that simply characterizing grounding as the relation with the help of which the world's inhabitants are hierarchically structured, is enough to, with justification, attribute to grounding most of the (formal) properties that have normally been taken to characterize it. Whether or not grounding exists, as well as what nature it has if it does, are however topics for another paper.

Both grounding and metaphysical explanation warrant our continued close attention. In order for the debate on grounding and on metaphysical explanation to progress in an orderly fashion, however, I suggest those investigations be conducted separately.

Acknowledgements The ideas presented in this paper evolved slowly and with much appreciated help from various audiences around the world, including, but not limited to, audiences at the 2014 'Truthmaking as Grounding: For and Against' workshop in Barcelona; the 2015 'Grounding' workshop in Edinburgh; the 2016 Nordic Network in Metaphysics meeting in Agder; the 2017 Ranch Metaphysics meeting in Tucson; and the 2017 Metaphysical Explanation workshop in Gothenburg. I'm especially indebted to Johan Brännmark, Daniel Giberman, Petter Sandstad, Jonathan Shaheen, Alexander Skiles, Ylwa Sjölin Wirling, Naomi Thompson, and an anonymous reviewer from this journal for thought provoking comments and suggestions. This research was completed with generous support from Riksbankens Jubileumsfond.

Open Access This article is distributed under the terms of the Creative Commons Attribution 4.0 International License (http://creativecommons.org/licenses/by/4.0/), which permits unrestricted use, distribution, and reproduction in any medium, provided you give appropriate credit to the original author(s) and the source, provide a link to the Creative Commons license, and indicate if changes were made.

\section{References}

Audi, P. (2012a). Grounding: Toward a theory of the in-virtue-of relation. The Journal of Philosophy, $109,685-711$.

Audi, P. (2012b). A clarification and defense of the notion of grounding. In F. Correia \& B. Schnieder (Eds.), Metaphysical grounding (pp. 101-121). Cambridge: Cambridge UP.

Bernstein, S. (2016). Grounding is not causation. Philosophical Perspectives, 30(1), 21-38.

Bliss, R. (2013). Viciousness and the structure of reality. Philosophical Studies, 166(2), 399-418.

Bliss, R. (2014). Viciousness and circles of ground. Metaphilosophy, 45(2), 245-256. 
Cameron, R. (2008). Turtles all the way down: Regress priority and fundamentality. The Philosophical Quarterly, 58(230), 1-14.

Cameron, R. (2016). Do we need grounding? Inquiry, 59(4), 382-397.

Clark, M. J., \& Liggins, D. (2012). Recent work on grounding. Analysis, 72(4), 812-823.

Correia, F. (2010). Grounding and truth-functions. Logique et Analyse, 53, 251-279.

Daly, C. (2005). So where's the explanation? In H. Beebee \& J. Dodd (Eds.), Truthmakers (pp. 85-103). London: Routledge.

Dasgupta, S. (2014). On the plurality of grounds. Philosophers' Imprint, 14(20), 1-28.

Dasgupta, S. (2017). Constitutive explanation. Philosophical Issues, 27, 74-97.

deRosset, L. (2010). Getting priority straight. Philosophical Studies, 149(1), 73-97.

Fine, K. (1995). Ontological dependence. Proceedings of the Aristotelian Society, 95, 269-290.

Fine, K. (2012). Guide to ground. In F. Correia \& B. Schnieder (Eds.), Metaphysical grounding (pp. 37-80). Cambridge: Cambridge UP.

Friedman, M. (1974). Explanation and scientific understanding. Journal of Philosophy, 71(1), 5-19.

Hempel, C. (1965). Aspects of scientific explanation. New York: Free Press.

Hempel, C., \& Oppenheim, P. (1948). Studies in the logic of explanation. Philosophy of Science, 15, $135-175$.

Hofweber, T. (2009). Ambitious, yet modest, metaphysics. In D. Chalmers, D. Manley, \& R. Wasserman (Eds.), Metametaphysics (pp. 260-289). Oxford: Oxford UP.

Khalifa, K. (2013). The role of explanation in understanding. British Journal for the Philosophy of Science, 64(1), 161-187.

Kim, J. (1988). Explanatory realism, causal realism and explanatory exclusion. Midwest Studies in Philosophy, 12(1), 225-239.

Kim, J. (1993). Postscripts on supervenience. In Supervenience and mind: Selected philosophical essays (pp. 161-74). Cambridge UP.

Kim, J. (1994). Explanatory knowledge and metaphysical dependence. Philosophical Issues, 5, 51-69.

Kment, B. (2014). Modality and explanatory reasoning. Oxford: Oxford UP.

Koslicki, K. (2015). The coarse-grainedness of grounding. In K. Bennett \& D. W. Zimmerman (Eds.), Oxford studies in metaphysics (Vol. 9, pp. 306-342). Oxford: Oxford UP.

Koslicki, K. (2016). Where grounding and causation part ways: Comments on Schaffer. Philosophical Studies, 173(1), 101-112.

Kovacs, D. M. (2017). Grounding and the argument from explanatoriness. Philosophical Studies, 174(12), 2927-2952.

Miller, K., \& Norton, J. (2017). Grounding: It's (probably) all in the head. Philosophical Studies, 174(12), 3059-3081.

Paul, L. A. (2012). Metaphysics as modeling: The Handmaiden's tale. Philosophical Studies, 160, 1-29.

Raven, M. J. (2013). Is ground a strict partial order? American Philosophical Quarterly, 50(2), 191-199.

Raven, M. J. (2015). Ground. Philosophy Compass, 10(5), 322-333.

Rodriguez-Pereyra, G. (2005). Why truthmakers. In H. Beebee \& J. Dodd (Eds.), Truthmakers (pp. 17-31). Oxford: Oxford UP.

Rodriguez-Pereyra, G. (2015). Grounding is not a strict order. Journal of the American Philosophical Association, 1(3), 517-534.

Rosen, G. (2010). Metaphysical dependence: Grounding and reduction. In B. Hale \& A. Hoffman (Eds.), Modality: Metaphysics, logic, and epistemology (pp. 109-135). Oxford: Oxford UP.

Salmon, W. (1984). Scientific explanation and the causal structure of the world. Princeton: Princeton UP.

Schaffer, J. (2009). On what grounds what. In D. Chalmers, D. Manley, \& R. Wasserman (Eds.), Metametaphysics (pp. 347-383). Oxford: Oxford UP.

Schaffer, J. (2016a). Grounding in the image of causation. Philosophical Studies, 173, 49-100.

Schaffer, J. (2016b). Ground rules: Lessons from Wilson. In K. Aizawa \& C. Gillett (Eds.), Scientific composition and metaphysical ground. Basingstoke: Palgrave MacMillan.

Strevens, M. (2008). Depth: An account of scientific explanation. Cambridge: Harvard UP.

Strevens, M. (2013). No understanding without explanation. Studies in the History and Philosophy of Science, 44, 510-515.

Thompson, N. (2016). Grounding and metaphysical explanation. Proceedings of the Aristotelian Society, 116(3), 395-402.

Trogdon, K. (2013). An introduction to grounding. In M. Hoeltje, B. Schnieder, \& A. Steinberg (Eds.), Varieties of dependence (pp. 97-122). Munich: Philosophia Verlag. 
Trogdon, K. (forthcoming). Grounding-mechanical explanation. Philosophical Studies. https://doi.org/10. 1007/s11098-017-0911-8.

Waskan, J. (2006). Models and cognition. Cambridge: MIT Press.

Wilsch, T. (2015). The nomological account of ground. Philosophical Studies, 172(12), 3293-3312.

Wilsch, T. (2016). The deductive-nomological account of metaphysical explanation. Australasian Journal of Philosophy, 94(1), 1-23.

Wilson, J. (2014). No work for a theory of grounding. Inquiry, 57(5-6), 535-579.

Wilson, A. (forthcoming). Metaphysical causation. Noûs. https://doi.org/10.1111/nous.12190.

Wittgenstein, L. (1953). Philosophical investigations. Oxford: Blackwell.

Ylikoski, J. (2009). The illusion of depth of understanding in science. In H. De Regt, S. Leonelli, \& K. Eigner (Eds.), Scientific understanding (pp. 100-119). Pittsburgh: U of Pittsburgh Press. 